\title{
Arthur und der Graal.
}

Meine Untersuchungen über die Anfänge der matière de Bretagne, die ursprünglich der Einleitung zum Perceval dienen sollten, sind längst über diesen Rahmen hinausgewachsen und müssen gesondert hervortreten. Der Streit der letzten Jahre dürfte gezeigt haben, da/s man ohne umfassenderes und eindringenderes Studium der Quellen nicht weiter kommen wird. Manche kymrische und selbst englische Hilfsmittel sind bisher vernachlässigt worden, Layamons Bericht über Arthur's Tisch z. B. wird zu Gunsten des Tischlein deck dich und ähnlicher Allotrien übersehen. Bei den lateinischen steht es noch schlimmer, hervorragend Wichtiges, wie Johannes Glastoniensis blieb ganz unbeachtet, nicht einmal Galfrid wurde genau angesehen. Hauptaufgabe der folgenden Artikel ${ }^{1}$ ist es, híer eine solide Basis zu schaffen; wenn auch nicht Alles eindringend genug behandelt werden kann, sollen wenigstens die Lücken deutlich werden. Endziel bleibt natürlich die Entwickelungsgeschichte der französischen Dichtung. Dals die Ergebnisse mit Zimmers Aufstellungen nicht übereinstimmen, mag von vorne herein gesagt sein. Mit der von Gaston Paris angekündigten Abhandlung über den gleichen Gegenstand werde ich wohl vielfach zusammentreffen, glaube aber nichts überflüssiges $\mathrm{zu}$ thun, wenn ich trotz dieser Voraussicht das Wort ergreife.

I.

\section{Wilelmi de antiquitate Glastoniensis ecclesiae.}

Als Holtzmann in der Germania 1867 , S. 257 ff. die Ansicht Nash's, dals Arthur den Kymren vor Galfrid unbekannt gewesen sei, weiter zu begründen suchte, stand ihm auch das Zeugnis Wilhelms von Malmesbury entgegen. Die Aechtheit der Stelle über Walwans Grab in den Gesta regum zweifelt er an, oder will sie erst nach Galfrids Buch geschrieben sein lassen, über das Buch de antiquitate Gl. eccl. sagt er (S. 276) "Jene Schrift ist allerdings von Wilhelm geschrieben und sogar noch vor den Gesta regum; aber in

1 Wilhelm von Malmesbury. - Geffrey Gaimar. - Arthur in Wales. Die runde Tafel. - Galfredus. - Vita Gildae. - Arthur in der Bretagne. - Die Lais. - Johannes Glastoniensis. - Perlesvaus und Robert. - Wolfram.

- Tristan. 
der Hs. des Werkes, welche bei Gale abgedruckt ist, werden die Namen der Aebte bis zum Jahr 1234 gegeben. Es ist darin von mehreren Schenkungen Arthurs die Rede und von seinem Grab, von welchem man vor 1193 nichts wusste. Es ist also deutlich, dals die Schrift Wilhelms im 13. Jahrh. überarbeitet wurde, und es versteht sich von selbst, dals man im 13. Jahrh. den Gaifrid und die späteren Romane lesen konnte".

Von Holtzmanns Aufstellungen sind mehrere sehr unvorsichtige, wie die über die Abfassung der Annales Cambriae im 13. Jahrh., inzwischen vom Schauplatz verschwunden, andere spielen in etwas modifizierter Fassung noch ihre Rolle in dem Kampf um Kaerleon. Seine Beurteilung der Klostergeschichte wurde dagegen allgemein rezipiert, halb und halb auch von dem namhaftesten oder einzigen namhaften Vertreter der wälschen Tradition, Gaston Paris. ${ }^{1}$ Allerdings ist es unzweifelhaft, dafs in De ant. eine Hand des I3. Jahrh. sich deutlich erkennen lärst, es hätte selbst die Frage aufgeworfen werden dürfen, ob Wilhelm von Malmesbury wirklich ihr Verfasser ist, ob die Stellen der Gesta, welche auf jene hinweisen und deutlich den Charakter zum Teil widerspruchsvoller Zusätze tragen, nicht selbst interpoliert seien. Die Neuausgabe der Gesta von Stubbs (London 1889) läfst indessen über die Authenticität keinen Zweifel. Für den Literarhistoriker ist damit die Aufforderung gegeben zu untersuchen, welche Teile der Klosterchronik jüngeren Datums sind und welche nicht, wie das bei jedem interpolierten historischen Denkmal zu geschehen hat, ehe man eine oder die andere Nachricht als Interpolation bezeichnet. Es wird sich dabei herausstellen, dafs wir auch ohne die Hilfe der Gesta längst dazu im Stande gewesen wären: aber Schwierigkeiten, wie die genannten, sind geeignet, den prüfenden von vorne herein abzuschrecken, und mögen die Ursache gewesen sein, dals eine der wichtigsten Urkunden zur Geschichte der Arthursage bisher fast ganz vernachlässigt wurde.

Wilhelm stand bis 1125 in keinerlei näheren Beziehungen zu Glastonbury; in den Gesta pontificum wie in der ersten Redaktion der Gesta regum spricht er wenig freundlich von der Abtey; später redet er von ihren Mönchen in Ausdrücken, die mindestens eine Gebetsbruderschaft bezeichnen, steht in näherer Beziehung zum Abte Heinrich von Blois ( I I 26-7 I), ändert sein historisches Urteil über die dortigen Traditionen, aus welchen er die Neuausgaben der Gesta ergänzt, verfalst mehrere Vitae der Ortsheiligen, und die Schrift De Antiquitate, welche unmittelbare Benutzung des Klosterarchivs voraussetzt. Er hat ohne Zweifel (cf. Stubbs 1. c. XXIX) längere Zeit an Ort und Stelle geweilt. De Ant. ist Heinrich von Blois nach dessen Ernennung zum Bischof von Winton gewidmet, also nach 1 I 29 geschrieben, vor 1139 , da er diesen weder zu Ein-

1 Entschieden Rom. X, 49I, zweifelhaft Hist. lit. XXX, I99. Rom. XVIII, $5^{89}$ ist gegenüber Zarnckes Angriff auf die Tradition von Joseph von Arimathia neue Untersuchung der Frage gefordert. 
gang noch am Schlusse legatus nennt, wie er von diesem Datum $a b$ in der Fortsetzung der Gesta regelmäfsig that, und vor II 35, weil er bei Angabe der Verwandtschaftsbeziehungen des Abts Heinrich I., Thibaut v. Blois und Adele nennt, aber nicht Stephan.

In Widerspruch mit dieser Zeitbestimmung stellen sich nun

a) Eine Abtliste (Migne 1725, Hearne 103), 1 welche bis 1234 geführt ist, in drei Columnen Jahrzahl, Namen und Regierungszeit angiebt, sehr ungeordnet, mehrfach in Widersprüchen mit sich selbst sowohl als mit Wilhelm. Die Stelle an der wir sie finden, ist eine ganz ungehörige; Wilhelm hat sich in der Klostergeschichte beim letzten ags. Abt unterbrochen, um in Aufzählung der aus dem Convent hervorgegangenen Bischöfe, der Donationen und einem Besitzverzeichnis einen Rückblick auf den alten Glanz des Hauses zu werfen. Zwischen hinein ist das Abtverzeichnis unvermittelt eingeschoben: es stand am Rand der Vorlage, ein Leser hatte es, angeregt durch das noch auf derselben Rückseite folgende Kap. De Turstino primo abbate de Normannis dorthin geschrieben, und der Kopist mechanisch einbezogen.

b) Die Translatio und revelatio Dunstani Hearne $3 \mathbf{I}-38$, Migne 1694-97. Die letztere schliefst sich an den grofsen Klosterbrand von II 84, und die Erzählung kann noch beliebig jünger sein: der Schlufssatz ,ibidem non cessat sua operari magnalia" wird nicht unmittelbar nach der Auffindung geschrieben sein. Die Fabel der translatio hängt enge mit der revelatio zusammen, ist für sie erfunden: Wilhelm in seiner mit De antiquitate gleichzeitigen Vita S. Dunstani (in Stubbs, Memorials of St. Dunstan) kennt nur die Beisetzung des Heiligen in Canterbury, deutet mit keinem Wort Ansprüche von Glastonbury auf die Reliquien an. Der Interpolator schrieb bei dem unmittelbar vorausgehenden Kapitel $D_{e}$ diversis reliquiis Glastoniae repositis sein Sanctus Dunstanus, cujus translationem a Cantuaria ad Glastoniam subjungemus, und der folgende Zusatz verrät sich schon durch die ungeeignete Stelle: er würde nach dem Aufbau des Buches dem Restsummarium der Reliquien vorausgehen müssen. Die weiteren Erwähnungen Dunstans, Hearne S. 7 I u. 92, sind dagegen unverändert in der Fassung Wilhelms belassen.

c) Im erwähnten Reliquienverzeichnis sind auch die Namen der Ursula und Daria zugesetzt, welche nach Johannes Glastoniensis S. I67 erst von Heinrich v. Blois gegeben waren. Wilhelm würde diesen Umstand erwähnen. Die beifügende Hand ist sicher die gleiche.

1 Abgedruckt ist De ant. bei Gale, Quindecim Scriptores, S. 289 und von Hearne im ersten Bd. seiner Ausgabe des Adam v. Domerham, reprodu. ciert nach Gale von Migne, Patr. lat. II, 179, Sp. 1689. Ich citiere nach Hearne, der die bessere Hs. benutzt hat, füge aber, so wẹit das nützlich ist, die Migneschen Spaltenzahlen bei. 
d) H. S. 26, M. 1691, De reliquiis s. David wird von diesen gesagt, dals quidam affirmant, sie seien nach Glastonbury gebracht worden; die Angaben der Wallenses über vergebliche Nachforschungen in Rosnaeum bestätigen das. Was als Schlufs des Kapitels folgt mit dem anschliefsenden kleinen Abschnitt $D e$ reliquiis a Guallia Glastoniam translatis giebt sich schon durch die einleitenden Worte als Zusatz zu erkennen "Sed qualiter de Rosina valle usque ad Glastoniam dictae reliquiae translatae fuerint subjungemus" variirt dabei das Zeugnis der Wallenses (cf. Joh. Glast. S. I 30). In den Auszügen aus De Antiquitate, welche Wilhelm der dritten Redaktion der Gesta regum einfügt, findet sich denn auch I, 25 die unerweiterte ursprüngliche Angabe.

e) König Heinrich wird S. I I6 u. I 20 (I73 I u. 33) als primus bezeichnet. Die Stellen sind im Uebrigen alt, die unterscheidende Zahl zu irgend einer Zeit nach II 50 beigesetzt.

f) S. 40 (1698) beginnt das Kapitel De altari Sancti David, quod dicitur vulgo Saphirus mit den Worten: legitur in vita Sancti David, Menevensis archiepiscopi, schlielst damit wie "piae recordationis Henricus Wintoniensis episcopus" den Altarstein wiederfand und ihn schmückte sicut adhuc apparet. Der Abschnitt ist also nach dem Tode Heinrichs ( I I 7 I) geschrieben, die Ausdrucksweise deutet auf eine nicht unerheblich spätere Zeit, kann selbst aus der Vita stammen, aus der auch die Erweiterung d herrührt.

Diese Interpolationen haben den ursprünglichen Text nicht alteriert, wie besonders bei d hervortritt; wenn die Abtsliste (a) mehr zufällig vom Rande hereinkommt, die revelatio (b) gewollter Zusatz ist, so giebt diese sich doch zugleich unverhohlen als solchen zu erkennen. Nach absichtlichen Fälschungen, etwa im Zusammenhang mit den späteren Besitzstreitigkeiten der Kirche, habe ich vergeblich gespürt. Die Ausdrucksweise pafst durchweg zu der Zeit, in welche die an erster Stelle besprochene datirbare fallt, beziehungsweise zu jener der zweiten, die als Anhaltspunkt vielleicht vorzuziehen ist, da sich die Abtsliste allzu leicht verlängern mochte. Eine gewisse (ileichartigkeit tritt auch darin hervor, dafs a gleich $b$, $d$ und $f$ mit Ueberschrift versehen ist. Wahrscheinlich sind also alle gleichzeitig. Nach ihrer Entfernung erscheint die Schrift in Aufbau und Rückweisen - mit einer unten zu besprechenden Ausnahme - klar und einheitlich: Die wesentliche Authenticität wird aufserdem durch den Vergleich mit den Gesta regum und der Vita Dunstani bestätigt. Trotzdem müssen wir, da nun einmal Einschiebungen stattgefunden haben, jede für die Arthurfrage wichtige Mitteilung auf ihre Ursprünglichkeit untersuchen.

A) Erste Kirchengründung durch die Apostelschüler unter Joseph. S. 4 (1683). Wilhelm schrieb in den Gesta Pont. II, 91 nach den Anglo-Sax. Chron. die Gründung Glastonbury's Ini zu; was über die ältere Anwesenhenheit des h. Patricius verlautete teilt er mit, nicht ohne ein „si credere dignum" vorauszuschicken. An Ort und Stelle lernte er durch eine (gefälschte) Carta Patricii (I9) 
die von mündlichen Zeugnissen unterstützt wurde (22) ${ }^{1}$ eine viel weiter zurückgehende Vorgeschichte des Klosters kennen: schon 12 Schüler der Apostel Philippus und Jacobus hatten dort auf Gottes Befehl eine Marienkirche gebaut. Man brachte sie in Verbindung (6; vgl. S. 20) mit den 12 Hyden, die das Kloster in Glastonbury selbst besafs. Eine Erleuchtung für unsern Autor war es, dafs er zugleich in dem ihm früher ebenfalls unbekannten Freculf 2 die Notiz über die Predigt Philipps in Gallien fand (5): von ihm also waren offenbar die Sendboten nach Britannien geschickt. Dafs Joseph sie hinübergeführt habe bezeugte anscheinend nur mündliche, jedenfalls eine nicht genügend gesicherte Tradition: quibus ut ferunt, carissimum amicum suum Joseph ab Arimathia, qui el dominum sepelivit, praefecit. Das Bedenken wurde nicht entfernt durch illae.... litterae apud St. Edmundun repertae (S. I I), nach welchen ecclesiam Glastoniae .... ipsi discipuli Christi.... aedificaverunt; die Stelle wird vielmehr ausdrücklich auf die Aposteljünger bezogen, und in demselben Sinn mufs daher die ungenaue Bezeichnung der "Christusschüler" S. 9 und Gesta Rg. I, I 9 verstanden werden. Die S. 30 ausgesprochene Ueberzeugung, dafs die Zwölfe im Kloster begraben seien, konnte sich also nicht auf Joseph erstrecken, ${ }^{3}$ noch weniger durfte dieser in den kurzen Excerpten der G. R. Platz finden.

Wir können in den Hauptpunkten ziemlich genau verfolgen, wie die Fabelbildung das Alter des Klosters immer weiter zurückgeschraubt hat. Am nächsten lag es den h. Patricius, als einen Wälschen, zu annektieren; man liefs ihn in das Vaterland zurückkehren. Beda's Angabe über eine Missionierung Britanniens durch Papst Eleutherius hatte Anlafs gegeben, die Namen der Sendboten Phaganus und Deruvianus ${ }^{4}$ irgendwie beizubringen, sie finden sich unabhängig von Wilhelm bei Galfr. Monumet IV, 19 (Faganus et Duvianus, mit Verlust des Abkürzungszeichens) und in veränderter Form im Liber Landavensis S. 65 (Eluanus und Meduuinus, waren wie gemacht, um annektiert zu werden. Aber die älteste Kirche sollte göttlichen Ursprungs sein (wie Monte Gargano von St. Michael gegründet war) oder doch wenigstens auf die Schüler Christi zu-

1 Vielleicht auch noch durch einige nicht benannte Schriftstücke. Der unter dem Abt Johann de Tantonia (vor 1290) gefertigte Index cartarum des Klosterarchivs (s. in Hearnes Ausg. des Joh. Glastoniensis S. $370 \mathrm{ff}$.) führt als Indulgencia in carta sine sigillo an: Eleutherius papa dedit $X$ annos indulgenciae, impetrantibus Phagano et Deruviano (Vgl. S. 20 der Charta Patricii) Caelestinus $p$. dedit XII annos, s. Patricio impetrante. Item Sti. Phaganus et Deruv. adquirebant $X X X$ annos. Mehrere Einzelheiten in D. A., insbesondere die Jahrzahlen, könnten hier entnommen sein. zeichnct.

2 Im Bücherkatalog des Klosters von 1247 sind zwei Exemplare ver-

3 Selbstverständlich ist übrigens, wenn Joseph die Jünger nach Britannien führt, damit keineswegs gesagt, dafs er bei ihnen geblieben und bestattet sei. Wir werden davon noch weiter zu sprechen haben.

1 Der eine klingt an den schottischen Heiligen Fagnanus an. 
rückgehen. 1 Den Anspruch zu fixieren diente Freculf II, 2, 4, nach welchem Jacobus Zebedaei Spanien und den Westländern, Philpp Gallien das Evangelium gebracht hätte; man liefs also ihre Schüler in der beliebten Zwölfzahl herüberkommen. ${ }^{2}$ Der lokale Ehrgeiz trifft hier mit der vielgestaltigen Tendenz wörtlichster Erklärung des docete omnes gentes zusammen. In dieser sehr bedenklichen Gesellschaft, die Wilhelm schon hier zu einigen verlegenen Wendungen veranlafst ${ }^{3}$ und an die er etwas später in G. R. I, I9 selbst nicht mehr recht glaubt - war Joseph jedenfalls der unsicherste Cantonist. Ich glaube, dafs er ganz willkürlich herbeigezogen wurde, nur weil er populär war und verfügbar schien: wie die späteren 12 von Phaganus und Deruvianus eingesetzt waren, ihre Nachfolger von Patricius reguliert wurden, sollten auch die ersten einen Leiter haben: es giebt genug derartige Fälle. Abgesehen von der späteren Weiterbildung der Glastoniensischen Tradition bieten die A. SS. Martii II, 507 ff. nur zwei jüngere Stellen, die ihn mit dem Occident in Zusammenhang bringen. Richer von Senones (-1 264) erzählt, dals man in Moienmoutier seinen Leichnam besessen und verloren habe; die Angabe dürfte altüberliefert sein, aber es wäre seltsam, wenn sich im entlegenen Glastonbury ein Nachklang derselben (Kombination mit Philippus) fände. Auf ihr oder gar auf Wilhelm dürfte es beruhen, wenn Julianus im 14. Jahrh. Joseph mit Jacob nach Spanien und von da nach Gallien kommen läfst. So naturgemäls die Art des Vortrags der Fabel in D. A. nach alledem erscheint hat gerade aus inneren Gründen Zarncke in Paul und Braunes Beiträgen III, $330 \mathrm{ff}$. die Aechtheit der Stelle in Abrede gezogen. Er legt sich die Entstehung des Buches dahin zurecht (S. 326 ff.), dafs es im Dienst der Politik der normannischen Herrscher, um die englische Kirche von Rom unabhängig zu stellen, deren apostolischen Ursprung habe nachweisen sollen, dafs Wilhelm ad hoc die Zwölf erfunden habe u. s. w. Wie aber wäre es denkbar, dafs der Verfafser, der seine Darstellung mit Ueberlegung und Berechnung entwarf, gerade das willkommenste Mittelglied, den einzigen den er mit Namen nannte, nur an jener einen Stelle einführti, um ihn dann so ganz fallen zu lassen? 4

1 Nach den oben erwähnten litterae apud St. Edmundum repertae S. 11 muls auch S. 6: unus Britonum historiographus, prout apud St. Edmundum itemque upud St, Augustinum Anglorum apostolum vidimus als Ortsbezeichnung gefafst werden Er hätte sich sonst an das Buch St. Augustins bei Layamon ed. Madden 2, 23 bezw. 3, I erinnern lassen.

2 Wilhelm ist es nicht $z$ verübeln, wenn er die Quelle der Fälschung als Beleg für ihre Wahrheit benützt. Er stellt Philipp voran, unter dem Einflufs von Freculfs "Gallis praedicat Christum, barbarasque gentes vicinasque tenebris et tumenti Oceano conjunctas ad scientiae lucem deducit" läfst aber S. 53 auch wieder Jacob gelten, entsprechend der Charta Patricii. Die Kapitelüberschrift S. 4 ist also nicht interpoliert, wie Heinzel, Gralromane, S. 42 meint.

3 Die Ilaupturkunde, die charta Sti. Patricii, war trotz der scriptura vetustissima (S. 22) nach dem citierten Index des Archivs nicht signiert.

- Heinzel, der sich Ueber die fr. Graalromane S. 44 ebenfalls gegen Zarncke ausspricht, hat diesen nicht richtig verstanden; übersieht aufserdem dafs, was er selbst als Möglichkeit construiert, in dem fertur enthalten ist. 
Diese ganze Deduktion ist einer der vielen Belege dafür, wie heute noch die hervorragendsten Gelehrten, sobald es sich um kirchliche Dinge handelt, mit der Aufklärungs-Kritik des vorigen Jahrhunderts ins Blaue hinein operieren. Dals König Stephan, der Schützling Innocents V., der Ursächer sein soll, möchte noch als ein schwerer lapsus calami hingehen. Heinrich I., der zu nennen war, hat allerdings mit Rom in Streitigkeiten um das königliche Investiturrecht gelebt, wenn man dafür aber ohne Weiteres einsetzt „den Wunsch, zur Erhöhung des königlichen Ansehens die englische Kirche von Rom unabhängig zu stellen", so heifst das moderne Auffassungen den realen Thatsachen substituiren. Wilhelm selbst stand, wie bei dem Klostergeistlichen zu erwarten ist, nicht auf Seite des Königs: vgl. z. B. Gesta Regum V, 417. Für den ganzen Streit war aber ein Buch wie De Ant. vollständig gleichgiltig, auch wenn es den Apostel Philipp selbst einen Ausflug nach England machen und es bekehren liefs. ${ }^{1}$

Die Frage „konnte Joseph eigentlich ein Schüler des Apostel Philippus genannt werden?" erledigt sich einfach dadurch, dafs Joseph hier wie zum Teil auch in der späteren Glastoniensischen Tradition als 13 ter, nicht als einer der Zwölfe gedacht ist. Dafs die "Glastonburger Chronik v. J. 1529 " nur die 12 Schüler des Philippus, nicht aber den Joseph erwähne, wäre bedenklich, wenn es sich nur wirklich so verhielte. Das Citat bei Usher Britt. Eccl. Ant. (S. Io der Ausg. v. 1687) spricht nicht von zwölfen, sondern von den Apostelschülern schlechthin. Gemeint ist die bei Hardy, Descr. Catal. III, I 50 näher beschriebene Hs., welche übrigens bis I 264 geht. Ihre ersten Blätter fehlen jetzt, der erhaltene Text des Anfangs beginnt mit Ad adventum Anglorum CCLXXX: ist keine Erzählung, sondern eine sehr knapp gehaltene chronologische Berechnung, entsprechend den Charakter von Ushers Citat. Dafür sind am Ende mehrere Kapitel aus De Ant. ausführlich wiedergegeben, darunter gerade die oben unter $b$ und $f$ besprochenen Interpolationen. Da die Chronik also eben unsern interpolierten Codex benutzte, ist sie für die Textkritik vollständig wertlos.

Es ist fast unnötig noch darauf hinzuweisen, dafs bei Ausmerzung des bestrittenen Passus, die den vorausgehenden und folgenden Satz einleitenden igitur zu nahe auf einander folgen, und dafs die erwiesenen Interpolationen weit entfernt sind von der echt Wilhelmischen Vorsicht, die sich in dem fertur ausspricht.

B. Ineswytrin, Glasinsel, eine Benennung die als scheinbare Uebersetzung des englischen Glaston Bedenken erweckt hat. Sie ist sicher viel älter als Wilhelm, findet sich 5, 17, 19, 48, 63, 97. $(168387,88,1702,23)$ und Gesta reg. I, 27, 28. An den drei

1 Ich will doch nicht unberührt lassen, dafs eine Bekehrung überhaupt nicht stattfindet, der Barbarenkönig mit seinem Volk Heiden bleiben; das Leben der Jünger in Ineswitrin dient nur der Verherrlichung Glastonburys. Die Kirche in England wird auch hier erst durch einen Papst, Eleutherius, ins Leben gerufen. 
ersten und der letzten Stelle ist sie als das wälsche Wort für Glastonbury betrachtet, aus den drei anderen erhellt, dafs ein nicht mehr recht gebräuchlicher Flurname der nächsten Umgebung des Klosters vorliegt, der fünf Casaten oder Hiden umfalste. Insel heifst ein durch Gewässer und Sümpfe abgeschlossenes Gelände, wie die bekannte Isle of Ely, in der Nähe von Glastonbury Athelney, Michelney u. a.; so erscheint insula bei einer Reihe von Besitzungen des Klosters und bei diesem selbst gebraucht, die keltische Form erhält sich in dem Flurnamen Ineswerrth 98 (1724). Die Deutung des Adjektivs auf das Wasser quasi vitrei coloris circumfluentem bei Giraldus Cambr. Spec. Eccl. II, 9 (cf. Rom. XII, 5 ro) ist nur dann zulässig, wenn gläsern = grün gesetzt werden darf, andere Ortsnamen, wie Glasbury (cf. Itin. Kambriae I, 2, kaum nach der dort erwähnten grünen Färbung des Flusses, da diese nur gelegentlich eintritt), Glasgow (cf. Loth, Mabin, II, 256, sehr zweifelhaft), Kaer Wydyr (Taliessin XXX, 4, Eroberung Arthurs, kaum die Turris vitrea des Nennius, auch nicht $=$ Glaston, trotz Vita Gildae 10), wären heranzuziehen. Doch auch ein leuchtendes Fenster (vgl. Beda, Opera min., I 39; Wilelmi Gest. Pont. III, IOO) oder Schmuck aus Glasmosaik konnte die Benennung bestimmen. Jedenfalls ist sicher, dafs der Name mit Mythologie und Poesie nichts zu thun hatte. Aber er klang geheimnisvoll, barg eine poetische Anregung, die bei Chrétien wirksam geworden ist.

C. Avalloniae insula. 7, 9, 17, 22, 23, 46 (1685. 87, 90, A. B., 1701). Die dritte der Stellen ist für sich zu betrachten, an den übrigen ist der Name unlöslich mit der nächsten Umgebung verbunden und diese mit dem Ganzen. Ein Interpolator hätte vier mal Glastonia tilgen müssen, um Avallonia einzusetzen, und sich dabei vorsichtig an die ältesten Zeiten gehalten. So raffiniert aber fälschte man in jenen Tagen überhaupt nicht. "The Isle of Avalon" (rising with Glastonbury Tor at its highest point), so heilst noch heute der isoliert aus dem Alluvialland aufsteigende Landrücken: auch in Tor ist die ags. Form bewahrt.

D. Weltliche Sicdlung in Glastonbury und Erklïrung des Namens Avallon 16-i7 (1687). Das Kapitel Quomodo multitudo popularis primitus Glastoniam inhabitaverit kommt hier am besten vollständig zum Abdruck; mit Benutzung der nur ganz unerheblich von Hearne abweichenden Cambridger Hs. R. 5, 33 und von Add. 22934.

Descriptis fundacione, dedicacione ac postea invencione hujus oratorii, restat apponere qualiter haec insula a multitudine fuerit inhabitata. Legitur in antiquis Britonum gestis, quod a boreali Britanniae parte venerunt in occidentem duodecim fratres et tenuerunt plurimas regiones, Venedociam, Demeciam, Guther (Guthir C.), Kedweli, quas proavus eorum Cuneda tenuerat. Nomina eorum fratum inferius annotantur: Ludnerth (Ludnerch, Add.), Morgen, Catgur, Cathmor, Merguid, Morvined, Morehel (Moreel, Add.), Morcant, Boten, Morgent, Mortineil, Glasteing. Hic est ille Glasteing, qui per mediterraneos Anglos, secus villam quae dicitur „Escebtiorne“, scrofam suam usque ad Wellis, et a Wellis per inviam et aquosam viam, quae 
"Sugewege", idest scrofae via ${ }^{1}$ dicitur, sequens, porcellos suos juxta ecclesiam, de qua nobis sermo est, lactentem, sub malo invenit, unde usque ad nos emanavit quod mala mali illius „Ealdecyrcenas epple“ id est veteris ecclesiae poma vocantur: sus quoque "ealdecyrce suge" idcirco nominabatur, quae cum ceterae sues quatuor pedes habeant, mirum dictu, ista habuit octo. Hic igitur Glasteing, postquam insulam illam ingressus, eam multimodis bonis vidit afluentem, cum omni familia sua in ea venit habitare, cursumque vitae suae ibidem peregit. Ex cujus progenie et familia ei succedente locus ille primitus dicitur populatus. Haec de antiquis Britonum libris sunt.

Die Erzählung von der achtbeinigen Sau hat den ächten Erdgeschmack der inselkeltischen Schweinehirtensagen, und aus Nennius $\S 62$ ist bekannt genug, wie Cunedag mit seinen Söhnen, aus dem Norden von Manau Guotodin kommend, gegen $400 \mathrm{n}$. Ch. die Schotten aus Venedotien vertrieben haben soll. Auf den ersten Blick also erkennt man, dafs hier kymrische Ueberlieferungen wenigstens verwertet sind. Die „Bücher der Britonen“ geben aber noch mehr. In den von Loth, Mabinogion, veröffentlichten Genealogien aus dem Ende des IO. Jh. ist II, 323 gesagt, dafs der älteste Sohn Cunedas in Manau Guotodin zurückgeblieben war, und daran ist hier offenbar angeknüpft. Ebenda (319) finden wir die Namen der zwölf in einem Stammbaum vereinigt: $[L l]$ udnerth, ${ }^{2}$ map Morgen, map Catgur, map Merguid, map Moriutned, map Morhen, map Morcant, map Botan, map Morgen, map Mormayl, map Glast, unum sunt Glastenic qui venerunt que vocatur Loytcoyt (sic.) Die Identität ist vollständig, nur die Formen besser. Unser Autor sagt, dafs er die Namen an einer anderen Stelle fand, als seine übrigen Angaben (inferius annotantur), er hat also wahrscheinlich selbst die Nachkommen in Brüder verwandelt. $\mathrm{Ob}$ jene Genealogien gerade in der erhaltenen weiteren Gestalt benutzt sind, lärst sich nicht sagen, trotz der zweifachen Uebereinstimmung, von einem Zusammenhang Glasts mit Cunedda war jedenfalls in ihnen Nichts zu finden, auch nicht ausdrücklich gesagt, dals dieser von Norden kam.

In den Schlufsworten des Stammbaums wird Glastenic wohl nach Glasteing als Glasteinc zu lesen sein. Sehr nahe liegt es auch, das unverständliche Unum sunt in Unde sunt zu ändern, obwohl damit das Loytcoyt ${ }^{3}$ nicht klarer wird; vielleicht steht $u \bar{n}$ in der Hs. Dann aber wäre Glasteinc als Glasteinbiry zu fassen (lieber als in Glastenie o. Glasteniam zu ändern) und ergäbe sich, dafs der seltsame Eigenname Glast (anklingend in einer späten irischen Dichtung der Heldenname Eoho Glass) erst aus dem als Patronymicon gefafsten Glasting

1 Cf. terram quae sic appellatur Souuig, XII manantium. Donation Inis s. a. 725 in Kemble Cod. Dipl. LXXIV.

2 Nicht ,[J]udnerth".

8 Caer luit coyt in dem Nennius in einigen Hss. angehängten Verzeichnis der 28 britannischen Städte, nach Heinr. v. Huntingdon $=$ Lincoln, ebenso (Caer Liud Coit) Galfr. IX, 3. Lincoln pafst schlecht in den Gesichtskreis der Kymren. Thurneisen macht mich darauf aufmerksam, dafs Dradley in der Academy 1889, 305 Lichfield als die wirkliche Correspondenz nachgewiesen hat. Vgl. auch Loth, Des mots latins dans les langues brittoniques S. 18. 
erschlossen ward, wie bei Nennius 49, Glovi mit seinen Nachkommen aus Cair Glovi (Kaer Loyw', Gloucester, von lat. Glevum).

Der Name des Ortes erscheint bei Wilhelm und in den von ihm vorgefundenen älteren, gefälschten und ächten, Dokumenten noch in anderer Gestalt als das später ausschliefsliche Glastonia und Glastonbury. So 17 Glastonbiry, 67 u. 83 Glastingebiry, 62, 85 u. 87 Glastingabiry, 82 u. 86 Glastingebury, 70 u. 72 das Adj. Glastingensis. Das erste Privileg Inis bietet Glastingai $(52$, bei Kemble dafür Glastingaea in No. 51, aber Glastingei in 93), das magnum privilegium (56-58) Glastingeie (Kemble: Gilastingie), daneben Glasteie einmal als Nom. des Substantivs, zweimal als Genitiv des Femininadjektivs.

Glastinginsel ist also zweimal, Glastinsel dreimal belegt. ${ }^{1}$ Was das letztere bedeutet weifs D. A. gar nicht mehr, wir erfahren es erst durch die kymrischen Genealogien. Die Doppelform zeigt, dals vor Wilhelm und vor dem unbestimmbar älteren Privileg die Gründungsfabel vorhanden war, im selben engen Zusammenhang mit Wales. Den Anschlufs an Cunedag hat diese wohl erst in einem zweiten Studium gewonnen, im letzten sind die sehr überflüssigen Brüder hinzugetreten.

Nur ist es nicht Wilhelm selbst, der die Bücher der Bretonen benutzt hat. Das vorausgehende Kapitel Quomodo monachus quidam de Sanclo Dionisio de Glastonia referebat besagt, dals es selbst und das folgende (et hoc et quod subjungemus capitulum) aus der Epistola Gottfrieds, eines normannischen Mönchs von Glastonbury, entnommen sei. Die Zeitangabe für das von diesem erzählte Erlebnis, tempore $H$ [enrici] Blesensis abbatis ist auffallig, scheint zu besagen, dafs die beiden Kapitel erst nach dem Tode des Abts zugesetzt seien. Dem widerstreitet die redaktionelle Einrahmung des ersten sowohl ${ }^{2}$ als die Anfangworte des zweiten nicht unbedingt; auch der Zusatz b ist ähnlich angeschlossen. Denken wir uns indessen, dafs Wilhelm, als er im 7. oder 8. Jahr Heinrichs nach G. kam, den quidam monachus nicht mehr lebend fand, aus der epistola aber entnahm, dafs sie unter Heinrich und nicht unter dessen Vorgänger geschrieben war, so hat er sich von seinem Standpunkt aus ganz richtig ausgedrückt.

An den Abschnitt über die Besiedlung schliefst sich der etymologische De diversibus nominibus ejusdem insulae. Glastinbiry ist

1 Bei Kemble, Anglo-Saxon Charters, ist vor dem 11. Jh. Glastonia belegt nur in dem sicher gefälschten grolsen Privileg Inis No. 73, in No. 400 s. a. 944 und 567 s. a. 971 . Vor d. J. 944 steht Glasting - in 49, 51, 74, 76, 92, 93 , 178, 338, 101 7, 1050. Später im 10. Jh. noch in 406, 438, 525, 545, 569, 577 , $590,593,659,685$. Ohne daraufhin jene beiden Urkunden geradezu als unächt zu betrachten, dürfen wir jedenfalls Glasting- als die ältere Form bezeichnen; sie erscheint auch allein mit dem altertümlichen -biry =bury verbunden. Glaston- entsteht daraus durch Labialisierung des unbetonten Vokals, $-i m b$ zu omb, mit schriftsprachlicher Umdeutung auf das häufige -ton = town.

2 Ad comprobandam antiquitatem ecclesiae, de qua praefati sumus, paululum digrediamur - Sed haec hactenus. 
entweder englische teilweise Uebersetzung von brittisch Inswydrin (Vgl. Vita Gildae 10 u. 14) oder vom besagten Glasteing genommen. Den Namen insula Avalloniae hat entweder Glasteing gegeben, weil er die in jener Gegend seltenen Aepfel dort vorfand, und Aepfel brittisch avalla heirst: vel cognominatur de quodam Avalloc, qui ibidem cum suis filiabus propler loci secretúm fertur inhabitasse.

Nun ist Afaloc eine der ersten Erklärung entsprechende gut kymrische Ableitung von afal. Die Latinisierung Avallonia-Avallo aber würde der von ihr getrennten Deutung auf einen Mannesnamen entsprechen. Gerade die Konfusion zeigt das höhere Alter der Ueberlieferung. Afaloc war der alte wälsche Name. Die latinisierte Form ist schlecht, aber nicht schlechter als hundert ähnliche, Anlehnung etwa an den Namen der burgundischen Burg 1 und Grafschaft.

In dieser Form ist Isle of Avalon bis heute als Name der Bodenerhöhung hinter der Stadt geblieben; das ursprüngliche Verhältnis der drei Benennungen lälst sich kaum mehr ganz entwirren, weil Sümpfe und Wasserläufe, die das ganze Territorium umgaben und wohl auch coupierten, nach und nach trocken gelegt worden sind, und sich damit auch die Flurnamen verschoben. Auf das Vorkommen von ynis Afallach und Afailen für Glastonbury in der späteren wälschen Literatur lege ich kein besonderes Gewicht, sie können, wie Zimmer Zts. f. fr. Spr. XII, 247 will, gelehrt sein, eine Nachwirkung der Deutung in D. A., die auch Giraldus Cambrensis und Johannes Glastoniensis wiederholen, sie können eben so gut den dauernden Sprachgebrauch bezeichnen.

Die Aechtheit des Kapitels wird durch seine Kongruenz mit dem vorausgehenden und durch die Altertümlichkeit seines Inhalts gestützt, doch nicht völlig gesichert.

E. Arthurs Grab 43 (1 700). Das Kapitel De nobilibus Glastoniae sepultis dient als Einleitung zu dem folgenden De duabus pyramidibus; der Vergleich mit den nachträglichen Ergänzungen W.'s zu den Gesta regum iäfst keinen $Z$ weifel über seine Ursprünglichkeit:

Quantum autem Glastoniae ecclesia fuerit eciam primatibus patriae venerabilis et ad sepulturam desiderabilis, ut ibi potissimum sub protectione Dei genitricis, operirentur diem resurrectionis, multa sunt indicio quibus pro cautela fastidii abstineo. Praetermitto de Arturo inclyto rege Britonum, in coemeterio monachorum inter duas pyramides cum sua conjuge tumulato; de multis etiam Britonum principibus. Praetermitto etiam de Kenwino in una pyramide locato. Jnsuper tumulos regis Edmundi senioris in turri ad dexteram, Edmundi minoris ante magnum altare,
G. R. I, 2 I : ... Quantum vero is locus fuerit etiam primatibus patriae venerabilis, ut ibi potissimum sub protectione dei genitricis opperirentur diem resurrectionis, plura sunt documenta, quibus pro cautela fastidii abstineo.

ib. II, I 44 Communi ergo decretum consilio, et funus [Edmundi senioris] Glasto-

1 St. Ladre d'Avalon im Fabliau du prestre et du chevalier, Raynaud II, 47, ist Wallfahrtstätte, die Kirche noch vorhanden. Das bretonische Oertchen, mit welchem sich Zimmer Zts. f. fr. Spr. XII, 248 beschäftigt, ist doch wol zu unbedeutend, der Name auch nicht specifisch bretonisch, sondern einfach das alte Aballo. 
Edgari prius in capitulo ante introitum ecclesiae, modo in scrinio, quod etiam de martyre superbit Vincentio; de quibus si se locus dederit, non me ita frustra suscepisse causabitur posteritas. Taceo et episcoporum sepulcra Brithuvii et Brithuvoldi, quae porticum aquilonarem ad St. Johannem. Bapt. uberem insigniunt, Livingi etiam et Seifridi episcoporum, Alfari, Aedthelstani, Aethelwini, Aelnoti ducum; quorum videlicet ducum quilibet centum libratas terrae cum multis aliis bonis contulit Glastoniae.

In unserer Schrift selbst verweist überdies Wilhelm auf dieses Kapitel S. 75 (1714) bei dem Grabe Fdmunds mit den Worten sicut plus quam semel in hoc opusculo dixi d. h. 73 u. 43 (1 7 I 3 u. 1 700 ); 87 (17 I9) Sepultus est [Edgarus], ut praediximus, in capitulo ad hostium ecclesiae etc., ebenso wie er dort die Absicht ausspricht, weiter von ihnen zu reden. Wollten wir nur die Stelle von de Arturo-tumulato als interpoliert betrachten, so mürste auch das nächste etiam eliminiert werden, und dann, da das Praetermitto de multis Britonum principibus zu kurz wäre, um die Wiederaufname mit Praetermitto etiam zu gestatten, auch die Britonen und das zweite etiam, zugleich mit der S. 43 (I 701) wiederholten Erwähnung der bretonischen Fürstengräber. Nun würde Kenwin cf. 49 (1 702) fallen, weil das folgende Insuper eine längere Aufzählung voraussetzt, dann auch insuper. Der Fortgang von der ältesten zur späteren Zeit, welcher Wilhelms Kompositionsweise genau entspricht, wäre erst von dem Interpolator hergestellt: die Spur seiner Thätigkeit mit beispielloser Kunst verwischt.

Fin Grund zum Verdacht war allerdings gegeben. Wilhelm selbst sagte in der bekannten Notiz über die Auffindung von Walwens Grab, Gesta Regum III, 287: Sed Arturis sepulcrum nusquam visitur, unde antiquitas naeniarum adhuc eum venturum fabulatur. Warum hat er hier nicht geändert, wenn er später wirklich die Grabmäler in Glastonb. sah? Hier ist zu beachten, dafs die Revision der G. R. vorzugsweise dic beiden ersten Bücher betraf, und in diese die $\mathrm{Zu}$ sïtze aus $D_{e}$ antiquilate hereingenommen sind. Auch die Gesta pontificum sind noch in den letzten Jahren des Verfassers von ihm durchgesehen worden (ed. Iramilton S. XIV), die sachlichen und stilistischen Aenderungen zahlreicher als in den späteren Büchern der G. R. Trotzdem ist hier gerade das Kapitel De Glastonia II, 91 unberührt gelassen, obwohl die Tonart (nec situ nec amenitate delectabilis - semper post adventum Normannorum pessimis infracta rectoribus.s), der Z.weifel an der Echtheit der Patriciusreliquien (si credi fas est) und anderes mit D. A. im Gegensatz steht, und obwohl W. in der Vita Dunstani den Osbern scharf tadelt weil jener, gerade so wie hier noch er selber, König Ini die erste Klostergründung beimifst.

Ergab sich bisher kein Grund an der Aechtheit unserer Stelle zu zweifeln, so findet sie eine indirekte Bestätigung in den Berichten gerade über jenes Ereignis, das nach einer mehrfach ausgesprochenen Meinung ihre Einschiebung veranlafst hätte, die Ueber-

Zeitschr, f. rom. Phil. XIX 
tragung der Gebeine Arthurs unter dem Abt Heinrich von Sully. Wir besitzen darüber drei unabhängige Berichte: bei Adam von Domerham ed. Hearne S. 34 I (geschr. um I 300, nach älterer Klostertradition), in den Annalen von Margan s. a. I I9I (Annales monastici I, 2 I) und in Giraldus Cambrensis Specul. Eccl. II, 9: der letztere der ausführlichste und im wesentlichen genaueste. Die Zeit war nach den Ann. I 19I, nach Adam unwesentlich verschieden 648 Iahre nach Arturs Tod, d. h. I I go. Wie wir gesehen haben, deuteten die chronologischen Merkmale in den erweisbaren Zusätzen auf erheblich spätere Jahre, es lag zu ihrer Zeit Arthur nicht mehr zwischen den beiden Pyramiden, sondern in der Kirche begraben, und wir haben sie tendenzlos aufrichtig gefunden. Einer absichtlichen Fälschung, welche die Auffindung hätte vorbereiten sollen, entspricht die unzweckmälsige Kürze der Angabe nicht, entspricht es auch nicht, dafs die Berichte eine Berufung auf Wilhelm vermissen lassen. Adam verweilst nicht auf die versteckte Notiz in D. A., obwohl er gerade die erhaltene Hs. fortsetzte, und der Abt hat sie wahrscheinlich nicht gekannt. ${ }^{1}$ Dieser selbst zeigte Giraldus die - vorsorglich - im Grab gefundene Bleiinschrift, von ihm stammt also auch in der Hauptsache was unser Gewährsmann Näheres weils. Die Inschriften auf den Pyramiden waren unleserlich (ebenso die Annalen und quond a m nobiliter insculptas bei Adam), der Abt konnte zwar noch ein weniges entziffern, aber wesentlich kam seine Kenntnis vom König Heinrich Il, ${ }^{2}$ der ihm, seinem Verwandten, zu öfteren Malen von dem Grab in Glastonbury gesprochen hatte, anscheinend mit dem Hinweis auf die eines so grofsen Herrschers unwerte Ausstattung. Das Bleidokument mulste der Abt erfinden, um den entgegenstehenden Volksglauben der Walliser $\mathrm{zu}$ widerlegen, sonst fand man nur, was auf einem Kirchhof immer zu haben ist, einen tiefliegenden Holzsarg, Knochen, einen Zopf, den man der Königin zuschrieb und der bei Berührung zerfiel. Abt Heinrich fabricierte nicht mehr, als die Durchschnittsehrlichkeit eines überzeugten, aber vorsichtigen Klosterhäuptlings erforderte, und wenn er sich lieber auf die Angaben König Heinrichs berief, als auf seine zweifelhafte Lesung der Inschriften oder die Ortstradition erscheint er ganz verlässig: die konnten dritten gegenüber gar nichts nützén. Und es existiert noch ein direkter Beleg dafür, dafs Heinrich II. die Verbindung Arthurs mit dem Kloster gekannt hat: er nennt Arthur unter den Wohlthätern des Klosters in dem von ihm ausgestellten Privilegium, das Adam v. Domerham (S. 175) einreiht und das aufserdem innerhalb der Konfirmation und Erweiterung Heinrichs III. (ib. S. 419) erhalten ist.

Nun enthält freilich der Abdruck Hearnes und die Londoner

1 Neben der Verstecktheit der Notiz ist die Unordnung und zeitweilige Vernachlässigung zu berücksichtigen, die ein Ereignis wie der Klosterbrand für die Bibliothek notwendig nach sich ziehen mufste.

2 Giraldus folgerte irrtümlich, dafs die Translatio noch zu Zeiten Heinrichs II. erfolgt sei, der ein bis zwei Jahre vorher gestorben war. Dafs der König zugegen gewesen sei, wie ihm mehrfach unterstellt wird, hat er nicht gesagt. 
Hs. zwischen Britonum principibus und Praetermitto einen evident interpolierten Passus der bei Gale und in der Cambridger Hs. fehlt: Idem Arturus, anno incarnationis dominicae DXLII in Cornubia juxta Auvium Cambam a Modredo letaliter vulneratus est, qui inde, ad sananda vulnera sua, in insulam Avallonis est evectus, et ibidem defunctus in aeslati circa Pentecosten, fere centenarius aut circiter. Das ist fast wörtlich ${ }^{1}$ aus Galfr. XI, 2 entnommen. Zweifel an der Aechtheit des Kapitels könnte die Stelle nicht erwecken, sie unterbricht in ungehöriger Weise dessen Gefüge, kennzeichnet sich stilistisch unzweideutig als eine mechanisch einbezogene Randbemerkung: und da von ihr aus das Alter der Gräber nicht angefochten werden darf, bedeutet sie Nichts für die Differenzen zwischen D. A. und der Historia regum. Ich habe vorgezogen, sie oben ganz wegzulassen. Soweit die mir sonst noch vorliegenden Varianten ein Urteil gestatten, stehen unabhängig zwei gegen zwei Zeugnisse, ist dieser Zusatz eine jüngere Randglosse der gemeinsamen Vorlage. In der von Hearne benutzten Canterbury-Hs. sind in gleicher Weise noch eine Anzahl Galfridiana beigeschrieben.

Dafs nun aber Galfrid selbst rein zufällig den Namen Arthurs mit der insula Avalloniae verknüpft haben sollte, ist mehr als unwahrscheinlich; auch er kannte jene Tradition, mehr oder minder genau, und hat sie nach seinen Bedürfnissen verwertet.

F. Arthur und Ider 47, 96 (I 701. 23). In den Gesten Arthurs ist zu lesen, dafs der König Ider dem Sohne Nuts am Weihnachtstag in Kaerliun zum Ritter machte und ihn mit sich gegen drci bösartige Riesen auf dem Mons Ranarum, jetzt Brentecnol, 2 führte. Der Jüngling eilte den Begleitern heimlich voraus, überwand die Unholde, fiel aber vor Ermattung in Ohnmacht. So fand ihn Arthur, glaubte ihn todt, und mals sich die Schuld bei. In Glastonbury, wohin er darauf kam, setzte er 24 Mönche für dessen Seele ein, mit reichen Stiftungen an Land und Schätzen. So die erste der beiden Stellen, die sich in der Geschichte der Donatoren genau da befindet, wo sie hingchört: Dic drei Heidenkönige Lucius, die Acbte Patricius u. Benignus sind besprochen, von weiteren bretonischen Aebten weils man nur bei Worgret und zwei Nachfolgern die Namen: sed de his postea. Und nun folgt De illustri Arturo. Darauf die Schenkungsurkunde über Ineswitrin, welche den Namen Worgrets enthält, v. J. 601, und welche G. R. I, 27 wiederholt ist. Es würde bei dem Interpolator ungewöhnliches Geschick voraussetzen, dafs er sich so gemein zu geben wüfste, und wir mülsten ihm überdies wenig gemeine chronologische Kenntnisse zusprechen.

Nicht weniger fest eingefügt ist die zweite Stelle, zu Anfang des Besitzregisters: In primis rex Arturus tempore Britonum dedit Brentemaris, Poweldon, cum multis aliis terris in confinio sitis pro

1 Mit Hinzulügung des Alters Arthurs und des Pfingstfestes.

2 Bei Gale unrichtig Brentenol. 
anima Ider, ut supra tactum est, quas terras per Anglos tunc paganos supervenientes ablatas iterum post eorum conversionem ad fidem restituerunt, cum plurimis aliis, unde rex Domnoniae dedit terram Ineswitherin $V$. hidas. Auch hier sind Ausdrucksweise und Reihenfolge ganz so wie wir sie bei Wilhelm erwarten dürfen. Zweimal bringt das ein mittelalterlicher Fälscher gewils nicht zu Stande; er kann uns gerade durch seine Naivität täuschen, nicht aber durch eine Kunst, die seiner Zeit gegenüber vollkommen zwecklos war. Zweimal wird ihn auch der Zufall nicht so begünstigen. Zu bemerken ist, dals das Anrecht auf die beiden Besitzungen ein unbestrittenes war. Pultone und Brentemerse stehen im Domesdaybook als schon unter Edward registriertes Eigentum der Abtei.

Stünde die erste Stelle allein, so würde sie mir immerhin bedenklich gewesen sein. Nicht wegen des sagenhaften Inhalts. W. pflegt sich wol bei den Fabeln die er aufnimmt, wie der Geschichte von der Hirschkuh, die Kaiser Heinrich über den Flufs trug, durch ein ferunt o. ähnlich den Rücken zu decken, aber hier war ja der Riesenkampf durch eine Donation bestätigt, und dals es Riesen in entfernter Zeit gegeben habe, liefs sich füglich nicht in Abrede stellen, und schlimmsten Falls entschuldigte ja auch das "Legitur". Sehr auffällig sind dagegen die gesta ill. regis Arturi, die nicht etwa eine Randglosse sein können, da der abhängige Satz das vorausgehende "Arturi" fordert. Was sind sie? Anscheinend doch eine schriftliche Quelle; wenn W. sich auf sein Lesen beruft, hat er nicht blos eine Sage erzählen, oder ein Gedicht vortragen hören; er meint ein Buch, nicht den Vortrag oder das Büchlein eines Fahrenden, und seine Ausdrucksweise milst dem Buch eine gewisse Bedeutung bei. Zunächst wäre an einen der beiden Romane zu denken, welchen wir später in Glastonbury begegnen werden, das liber di gestis incliti regis Arturi. Dann allerdings wäre an der Interpolation nicht zu zweifeln, da der Inhalt dieses Buches (Verbindung einer eigenartigenartigen Lancelotsuche mit Gralqueste) einer erheblich jüngeren Zeit angehörte, auf den französ. Prosaromanen beruhte. Die erste Notiz über seine Existenz findet sich indessen erst Jahre nachdem unsere Kopie von De ant. geschrieben war, Die Identität der Namen hat wenig zu bedeuten, die altertümliche Form unserer Iderfabel palst schlecht zu jenen späten Erfindungen, und es läfst sich nicht recht absehen, wo sie innerhalb derselben ihre Stellung gefunden haben sollte: endlich scheint im franz. Ider nicht einmal der Name Lancelots zu stehen. Nehmen wir die oben entwickelten inneren Gründe für die Ursprünglichkeit unserer Episode hinzu, so murs jeder Zusammenhang in Abrede gestellt werden. In der Hist. lit. XXX, I $99 \mathrm{ff}$., wo allerdings der Gedanke an eine Interpolation überwiegt, ist ein anglonorm. Rittergedicht als Quelle angenommen. Eher als an ein episodisches Gedicht dürfte nach Zeit, Ort und Titel an eine Reimchronik gedacht werden, etwa an das später zu besprechende Buch Roberts von Gloucester. Vor jeder rein hypothetischen Quelle aber ist eine sicher benannte in 
Frwägung zu ziehen, um so mehr als Wilhelm sie benützt hat, die oben besprochene Epistola des Mönchs Gottfrid. Dort wie hier ist eine kymrisch-glastonsiensische Tradition erzählt, der Berufung auf die antiqua Britonum gesta, antiqui Britonum libri entspricht die auf die gesta regis Arturi. Beide Citate dürfen in anderem Munde weniger bestimmt gedacht werden, als in dem Wilhelms selbst.

Kymrisch-glastioniensisch ist die Idersage nach der Ortsangabe: in montem Rannarum, nunc dictum Brentecnol wie weiter hervorgeht aus 45 Bregden a quo Brentacnolle, quae nunc Brentamerse dicitur, vergl. mit 96 Arturus..., dedit Brentemareys, Poweldone. Die Sage war also bei einer Besitzung des Klosters lokalisiert und früher oder später mufste das dazu führen, auch Arthur mit ihm in Beziehung zu setzen. Die Angabe S. 45 ist nicht ganz genau, cnol Berg bezeichnet notwendig eine andere Lage als mersc, französisiert marais, mareis, und der letztere mufs an und nicht auf dem Mons Ranarum gelegen sein. Ein Acker der Besitzung lag am Axeflufs (S. 307), ein Teil scheint das Meer berührt zu haben, 2 eine Randbemerkung zu S. 47 setzt das umfassendere Brent dafür, und das Gleiche geschieht schon bei Wilhelm, wenn Ini S. 5 I u. 56 Brente $\mathrm{x}$ hidas, S. 97 Brentmareis $\mathrm{xx}$ hidas ${ }^{3}$ schenkt. Vgl. darüber die Enc. brit., Artikel Somerset: This plain, intersected by ditches... is broken by isolated hills and lower ridges, of which the most conspicuous are Brent Knoll near Burnham... Für den wälschen Pilger, der über den Bristolkanal nach Glastonbury kam, war der vereinzelte Berg die natürliche Landmarke. - Der kleine Widerspruch zwischen den nunc dicitur S. 45 u. 47 ist gerade bei einem Ortsfremclen nicht, auffallend, der zweierlei Quellen benutzt, wäre es übrigens nicht einmal bei einem einheimischen Mönch. Nur scheinbar widerstreitend ist es, wenn nach 96 Arthur, nach 97 Ini Brentemareis schenkt: nach dem Urkundenauszug S. 5 I hatte der erste angelsächsische Abt den Rechtstitel aufgegeben quam terram Berthzerald abbas sponte propria deseruit. So dient der letzte Zweifel nur dazu, den engen Zusammenhang der einzelnen Angaben zu dokumentieren.

Während Förster gerade die Iderfabel als kymrisch gelten liefs, dient Zimmer Zts. f. f. Sp. XIII, 22 auch sie als Zeugnis für die Arrnoricanertheorie. Edern kommt einmal als Ortsname in der Bretagne vor (cf. Loth, Mabin. I, 203), und aus dieser Form ward im Munde der romanisierten Bretonen Ider; warum, wird nicht gefragt. 4 Dafs der Held in dem so viel umfangreicher als das alt-

1 Wiederholt bei Johannes Glastoniensis, S. 32. In Gesta regum I, 2 I schrieb W. zutreffender: Bregden a quo Brentacnol et Brentemeirs.

2. 507: Maris acessum ibidem, quantum ad sortem suam pertinuit, multis sumptibus potenter obstruxit.

3 Die kleinere Zahl ist die der Urkunde Inis, die grölsere im Donationenregister giebt den Umfang der Besitzung gleichlautend $\mathrm{m}$. d. Domesdaybook.

4 Für Zimmer ist durchweg die unkeltische Form eines keltischen Namens ein unwiderlegbarer Beweis für den Durchgang durch die französische Bretagne. Er übersieht, dafs bei den Anglonormannen die Umgestaltungen wesentlich dieselben sein mufsten als dort. 
kymrische überlieferten altbretonischen Namenmaterial nicht fortlebt, ein Kriterium das sonst bei Z. eine so grofse und so ungerechtfertigte Rolle für die Bretagne spielt, bringt er hier nicht in Rechnung, weil es sich auch gegen die Bretagne kehren würde. In Wales scheint der Name als solcher nicht fortgelebt $\mathrm{zu}$ haben, gleich vielen anderen altberühmten; als Ortsbenennung findet er sich, wie in der Bretagne (Loth l. c., vgl. übrigens Edyrnioñ als Personen- und Ortsnamen im Rec. of Caernarvon), wir begegnen ihm aber alt noch bei einer zweiten Persönlichkeit der einheimischen Tradition, Etern dem Sohn Cunedas in den Genealogien der ältesten Hs. der Annales Cambrenses. Wir kennen die Idersage nur durch De ant. und die französische Dichtung. Galfrid X, 4, 5 hat den Namen in Verbindung mit Arthur, und die wallisischen Trümmer bieten auch nichts weiter von Edern ap Nudd, nur etwa, dafs ihn der späte Traum Rhonabwys zum Führer der Dänen macht. Der einfache und ursprüngliche Charakter der Erzählung Wilhelms wird Hist. litt. XXX, 200 mit Recht hervorgehoben. Dem thut nur wenig Eintrag, wenn sie von Verleihung der Ritterschaft spricht, einer Sitte, die den Kymren jener Zeit gewifs noch nicht geläufig war, obwohl sie sich später in dem Mabinogi von Math dem Sohn Mathonwys findet. In genauer Parallele mit ihr aber fand sich der alte Gebrauch des Haarscheerens, wie ihn Arthur in Kulhwch und Olwen (Loth, Mabinogion I, 2OI) übt, und es ist ganz natürlich, wenn in dem Latein des Anglonormannen Gottfried dafür insigniis militaribus decorare eintritt. Merkwürdig ist der Unterschied zwischen der literarischen Gruppe und dem Kymrischen in der Form des Namens: Edern, auch Edyrn auf der einen, Ider, bei Galfr. Hider auf der anderen Seite. Die Differenz im Anlaut dürfte auf kymrische Aussprache zurückgehen 1 (vgl. etwa Nettlau, Beiträge $\S 68$ ), nicht die im Auslaut. Als die in D. A. benutzte Autorität haben wir den Anglonormannen wahrscheinlich gemacht, für Galfrid deutet schon sein dem Ags. fremder Name ${ }^{2}$ auf dieselbe Nationalität, ebenso einige andere Indicien, wie Merlin f. Myrddin wegen merde. Die willkürliche Verwendung von jorn neben jor u. s. w. in den ältesten anglonorm. Denkmälern zeigt, dafs hier der Auslaut auch in Pausa und vorvokalisch gefallen war, jorn nur antiquarisierende Licenz ist. Wenn Gottfried und Galfrid den Namen schreiben wie ihn die Anglonormannen sprechen, deutet das auf mündliche Ueberlieferung, allgemeiner darauf hin, dafs einzelne kymrische Erzählungen unter den Eroberern in den Grenzdistrikten gehört wurden. Die Uebereinstimmung mag indes den Anlals geben, hier die Frage zu erörtern, ob nicht in De Ant. die Historia regum Britanniae benutzt ist, oder umgekehrt. Gemeinsam sind ihnen ja noch die Namen der Sendboten des Papstes Eleutherius und die Verbindung Arthurs mit Avalon. Für die Geschichte der Bekehrung des Lucius beruft sich Wilhelm (S. 9) neben der

1 Möglich ist auch Einwirkung des sehr üblichen Ithel (Juddhail).

2 Dessen Fälschung in "Gottfried“ endlich einmal verschwinden sollte. 
charta S. Patricii auf die Gesta Britonum, in welchen man Galfrid vermuten könnte. Da er aber so in den Gesta regum den Nennius bezeichnet, der $\S 22$ das Ereignis erzählt, muss dieser gemeint sein. Schon paläographisch ist ja der Name Duuianus bei Galfr. wahrscheinlich jünger als Deruvianus bei Wilhelm. Die Iderfabel erzählt G. überhaupt nicht, die Annahme der Beisetzung Arthurs in Glastonbury knüpft sich bei W. an die beiden Pyramiden und seine Einbeziehung der Königin pafst schlecht zu ihrer Flucht nach Caerleon bei G. Wir haben übrigens S. 328 Anlafs gefunden, De Ant. vor den Tod Heinrichs I. (i. J. I I 35) zu setzen, während die Schlufsworte in Galfrids Widmung entschieden auf die Zeit nach demselben hindeuten. Bei der Art, wie dieser mit seinen Quellen verfährt, könnte seine Angabe über Arthurs Fahrt nach Avalon immerhin durch W. beinflufst sein, trotz der Differenz bezüglich der Königin. Auch dafs er die Geschichte des Hider nicht erzählt, ist nicht zwingend. Stärker klingt die Geschichte der ersten Bekehrung an, wenn auch an Stelle der Besetzung Glastonburys die Einrichtung der Bistümer berichtet ist. Die fast wörtliche Uebereinstimmung zwischen

verbi dei incarnationem praedicantes, verbum vitae evangelizantes regem.. ipsum baptismate sacro abluerunt sacro fonte abluerunt

verdient einige Beachtung, der Gedanke, die zweie nach Rom zurückkehren und mit weiteren Gefährten wiederkommen zu lassen, könnte gerade durch die duodecim socii veranlafst sein. Nur auf diese beiden Texte gestellt, würde die Frage als offen bezeichnet werden müssen. Die dritte, erheblich abweichende Variante im Liber Landavensis ist entscheidend. Dort entspricht dem Verzeichnis der Archiflamines bei Galfrid ein ecclesiasticum ordinem constituit, episcopos ordinavit [sc. Lucius]; mit der wenig motivierten Rückkehr der Missionare nach Rom berührt sich, dafs sie hier ursprünglich die Boten sind, die Lucius dorthin schickt. So gewinnt die Angabe Galfrids an Glaubwürdigkcit, dafs er einen ausführlicheren Bericht in dem Buch gefunden habe quem Gildas de victoria Aurelii Ambrosii inscripsit. $\mathrm{Da}$ die sonst noch verwertbaren Natchrichten aus I) Ant., wie die von den Apostelschülern, St. Patricius, St. David, vollständig fehlen, mufs seine Benutzung im höchsten Grade unwahrscheinlich genannt werden.

Ich komme zum Schlufs. Es hat sich gegen keine der untersuchten Angaben ein ernstlicher Verdacht crgeben, bei der Mehrzahl lärst sich geradezu nachweisen, dals sie von Wilhelm selbst herrühren oder wenigstens, dafs sie älter sind als die datirbaren interpolierten Stellen. Die Tradition von Glastonbury stand in Sagen, Legenden, Gräbern, Reliquien in vielfacher Beziehung ${ }^{1}$ zum wälschen Nachbarland. Auf einer der ältesten Besitzungen des Klosters war die Iderfabel lokalisiert, Arthur ward daher als Donator betrachtet,

1 Mit Zimmers aprioristischer Läugnung des angelsächsisch-wallisischen Verkehrs werde ich mich späterhin auseiandersetzen. 
sein Grab unter die Gräber anderer wirklicher oder vermeinter Keltenfürsten auf dem alten Kirchhof verlegt. All das ist altertümlich, einfach, natürlich. Der vierte Artikel dieser Reihe wird zeigen, wie im letzten Viertel des 12. Jh. in Glastonbury eine fromme Arthurgeschichte erfabelt ist, welche die Romane, insbesondere den Perceval verwertete, Joseph und die klostergründenden Eremiten in höchst phantastische Beziehung zum Arturkreis brachte. ${ }^{1}$ Nichts von diesen Beziehungen, keine der dort in die Mttte gestellten Romanfiguren kennt De Ant. Noch etwas älter als jene Geschichte ist eine in ihrem Anfang verarbeitete Legende, deren Mittelpunkt ein von Arthur gestiftetes wunderbares Kristallkreuz bildet ${ }^{2}$ : bei der Ithersage ist dieses Kreuz unter den Schenkungen nicht genannt. Endlich haben wir gesehen, dafs sich nicht einmal Beziehungen $\mathrm{zu}$ Galfrid wahrscheinlich machen lassen. Von all dem würde sich doch irgend etwas Greifbares bei einem späten ArthurInterpolator finden.

Immerhin bleibt an einigen Punkten ein Zweifel an der Ursprünglichkeit möglich. Sicher dem Text Wilhelms gehören Joseph von Arimathia an, die Namen Ineswitrin und Avallonia.

Die gegen die Tradition von den Königsgräbern vorgebrachten Beweise haben sich haltlos gezeigt; sie ist sicher erheblich älter als die revelatio von IIgI, und lä/st sich aus dem Zusammenhang nicht auslösen. Um aber den Brief des Mönches Gottfried zu den Quellen Wilhelms rechnen zu können, wurde eine Hypothese notwendig, welche sich auf die gesta Arthuri erstreckt; sie wird noch weiter gestützt werden, bleibt aber darum Hypothese. Sicher ist bei Glast und der mit ihm verbundenen Namendeutung nur das Vorhandensein alter Ortssage kymrischer Herkunft, späteres Eindringen in D. A. nicht wahrscheinlich, aber immerhin möglich, und es mufs bemerkt werden, dafs die drei unter $D$ besprochenen, mit „digrediamur" eingeleiteten Kapitel sich ohne Schaden für den Zusammenhang streichen lassen. Die Idersage aus dem Text auszulösen scheint mir nicht erlaubt, obwohl es bequem wäre, das Kapitel De illustri Arturo mit der Gruppe D aus einer alten, aber Wilhelm fremden Quelle herzuleiten.

Ich hatte die vorliegende Untersuchung mehrere Jahre abgeschlossen liegen lassen, um sie auf die Handschriften zu prüfen: obwohl die Uebereinstimmung zwischen der Hs. Gales, Adam von Domerham und den unter A erwähnten Excerpten von vornherein wahrscheinlich machte, dafs im 13. Jh. im Kloster in Folge des Brandes nur eine Kopie existierte, eben die weiterhin mit den Randnoten versehene. Die erreichbare Sicherheit darüber verdanke ich nunmehr der bereitwilligen Hilfe der Herren Neubauer in Oxford, Birch in London, Rogers in Cambridge und besonders Rennert in Philadelphia.

1 Vgl. Ltbl. 1892. S. 160. abdruck.

2 Bei Johannes Glastoniensis I, 77; sie kommt im Folgenden zum Neu 
Von den bei Duffus Hardi II, 157 aufgeführten Mss. fallen hinweg Cott. Tiber A. (Adam v. Domerham); Ashmole 790 und Cambridge R. 5.16 (Johannes Glastoniensis); C. C. C. Cambridge IOI und Bodley, Wood (die Excerpte). Add. 22934 enthält den unter E besprochenen Zusatz Hearnes aus Galfrid nebst den sonstigen Erweiterungen, nur lärst es die von Gale, Hearne, den Excerpten und Cambridge gegebene Abstliste weg. Cambridge R. 5, 33 enthält sämtliche Erweiterungen, abgesehen von dem Zusatz Hearne: in beiden stehen alle angezweifelten Stellen. Die Abtsliste konnte beim Kopieren der Glastonburyhandschrift leicht in Wegfall kommen, selbst absichtlich, da sie offenbar etwas unbequem geschrieben war; auch für Add. lag sie schon vor, wie die Aufnahme einer jüngeren Beithat zeigt. Im Uebrigen sind die mir mitgeteilten Varianten wenig erheblich, sprechen gegen ein Filiationsverhältnis mit den Vorlagen der Drucke. Diesen selbst weiter nachzugehen lag kein Anlafs vor.

\section{II.}

\section{Das Buch Roberts von Glocester.}

Am Schlufs der Estorie des Engles spricht Geffrei Gaimar von seinen Quellen. Er hat sich manches Buch verschafft:

6443 Liveres engleis, e par gramaire

Et en romanz et en latin.

Die „manchen" sind dann freilich nur vier. Die estorie de Wincestre $(6468)$ hat er schon vorher mehrfach angeführt, an Stellen $(2234,2334)$ die mit voller Sicherheit in ihr die Anglo-Saxon Chronicles bezw. eine ihrer Formen erkennen lassen.

\section{De Wassingburc un livere engleis \\ $U$ il trovad escrit des reis \\ $E$ de tus les empereours \\ $K e$ de Rome furent seignurs \\ E d'Engleterre ourent treu}

ist nach dieser Inhaltsangabe der Orosius Alfreds. I Die lateinische Quelle war Galfredus Monumetensis:

$$
\begin{gathered}
0465 \text { Le bon livere de Oxi [ne] ford } \\
\text { Ki fust Walter l'arcediaen. }
\end{gathered}
$$

Gewifs hätte Geoffrei etwas genauer das Buch bezeichnen können, welches Galfrid von dem Archidiakonus Walter von Oxford erhielt, begeht er denselben Fehler, den die kymrische Uebersetzung zuigt und die mittelenglische des maister Gnaor, den auch noch andere Dilettanten gemacht haben: Aber darum ist die Sachlage doch nicht weniger klar. Ten Brink hatte verzeihlicher Weise Jhb. IX, 270 an eine vorgebliche Fortsetzung Galfrids durch Walter gedacht. Aber eine solche existiert nicht, Geoffrey hat nichts Der-

1 Kaum, wie Liebermann, D. Zts. f. Gesch. 7, E, 12 annimmt, noch einmal dasselbe mit der estorie de Wincestre. Was weiter folgt, beziehe ich auf den Inhalt der beiden Bücher. 
artiges benützt, und sagt qui fu, nicht que fist. Daran ändert Nichts, dafs Heeger, Trojanersage 83 diese Interpretation annimmt, ohne Rom. I, 45 I Widerspruch zu finden.

Französisch (en romanz) mufs also die vierte gewesen sein, welche die Auftraggeberin Dame Custance durch ihren Gemahl Raoul, den Sohn Gileberts, von Walter Espec entlieh, dem sie Graf Robert von Glocester gegeben hatte; Robert der Gönner Wilhelms von Malmesbury und Galfrids

\section{Fist translater icelle geste Solum les liveres as Waleis Qu'il aveient des bretons reis.}

Die ganz besondere Hervorhebung dieses Buches, die wahrscheinlich unberechtigte Bevorzugung gegenüber dem des Erzdiakons ist Folge seiner Beschaffung durch die Patronin. Weil Galfrid sein Buch demselben Robert widmet, geste und des bretons $r$ tis anklingt, zufällig auch noch der Walter Espec, hat man sich verführen lassen, hier Galfrid zu suchen, im klaren Gegensatz zu Wort und Inhalt.

Das Buch Roberts (gest. I I 46) dürfte im Rahmen der galloenglischen Literaturentwicklung als ein natürlicher Vorgänger von Davids Reimchronik Heinrichs I. erscheinen, die wir ebenfalls nur durch Gaimar ${ }^{1}$ kennen: Uebersetzungen vor der selbständigen Erzählung. $\mathrm{Zu}$ viel Gewicht dürfen wir auf dieses theoretische Verhältnis freilich nicht legen, müssen uns begnügen zu sagen, dafs Galfrid, David, und der unbekannte Kleriker ungefähr gleichzeitig sind. Ueber den Inhalt verrät Gaimar leider sehr wenig. Die einleitenden Verse seines allein erhaltenen letzten Buches greifen nur auf den Schlufs des vorausgehenden zurück, den Untergang des Brittenreichs, der, wie zu erwarten, auf Galfrid beruht: weiter erfahren wir nur noch (6530), dafs er mit dem Argonautenzug begonnen hat. Dafs Modret den Sachsen das Land Des Humbre trisk'en Cateneis gegeben habe (V. I I, cf. 4322), wovon Galfrid nichts weifs, wird persönlicher Rückschlufs sein: etwas auffälliger ist, wenn V. 6 Iwain König von Murray (Muref) und Lothian (Loeneis) genannt wird, statt von Albanien, aber auch dabei dürfte lediglich an das Frbe seines Vaters und Oheims gedacht sein. Einen Fingerzeig könnte die Berufung auf Gildas bieten, der zu Eingang der Havelokepisode 2 dafür angeführt wird, dals zur Zeit Constantins, des Neffen Arthurs, zwei Könige in Britannien gewesen seien. Gaimar war den ags. Chroniken gegenüber in grofser Verlegenheit, wie er Haveloc unterbringen sollte, die Autorität ist offenbar bei den Haaren herangezogen. Bei Nennius, der oft Gildas genannt wird, und an

1 Diese fortzusetzen hat er nicht beabsichtigt. V. $6485 \mathrm{ff}$. ist nach $6520 \mathrm{ff}$. zu beurteilen, eine scherzhafte Form für die Aufforderung an den lebenden David zur Vervollständigung des Buchs.

2 Es liegt keinerlei Grund vor, sie Waimar abzusprechen, ebensowenig als bei der Einleitung. Ich lasse die Vermutung bei Seite, dafs sich die Einleitung nicht auf das erste Buch Waimars beziehen möchte, sondern auf Wace, der dieses in den vier Hs. verdrängt hat. 
den man bei dem Buch Roberts denken dürfte, finden sich in den ags. Genealogien ( $\$ 57$ u. 6r) die beiden Königsnamen, aber ohne jeden Zusammenhang unter sich und mit Constantin, und weitere Vergleichung mit der Estorie zeigt zur Evidenz, dals Gaimar diese Listen nicht benutzt hat. Einige Citate bei Galfrid machen wahrscheinlich, dafs eine verlorene Schrift über Siege britischer Könige unter dem Namen des Gildas lief. Wenn aber der Name hier genannt ist, warum nicht auch 6449-92? Der Art gegenüber, in der sich Gaimar sonst mehrfach behilft, lälst sich überhaupt auf die Stelle nicht zu viel Gewicht legen, wir können aus ihr nur etwa die Möglichkeit entnehmen, dafs in der Kompilation aus den liveres as Waleis Gildas citiert war. - Wörtlich genommen sprechen $6452-53$ und 6462 von wälschen Quellen, und so unbestimmt, so nur annähernd genau die Angabe sein mag, dürfen wir nicht ganz verachten was geschrieben steht. In Gloucester 1 konnte man wallisische Ueberlieferungen auch unmittelbar beschaffen, mit den kambrischen Annalen oder der Historia Britonum verschmelzen. Es könnten die Geschichten des Geschichtsschreibers Istas darin stecken, die nach Brut y Tywysogion I I 97 berühmt waren.

Auf alle Fälle, und selbst wenn nur eine Nenniusredaktion im Hintergrund stünde, bleibt die Thatsache, dafs der französische Grofse im Grenzland für sich die Historien der britischen Könige, der Altvordern seiner Nachbarn, bearbeiten liefs. Eben in den Landstrichen, wo dieses Interesse am natürlichsten war, tritt es in der neu auflebenden Geschichtsschreibung Englands zu Anfang des zweiten Drittels des I 2. Jh. zu Tage: in den Gesta regum mit der Erwähnung von Gauvains Grab, dem Buch de antiquitate Glast. eccl., Galfrids Machwerk, und hier in Gloucester. Nicht die poetische, sondern die geschichtliche Anteilnahme überwog zunächst der Gestalt Arthurs gegenüber: erst später verfiel, was als Geschichte galt oder gelten sollte, der freieren bewufsten Fiction. In den Rahmen der ältesten franco-englischen gereimten Uebersetzungsliteratur pafst das Buch Roberts sehr gut herein; es veraltete schon durch Waimars Compilation, und verschwand mit dieser vor der Autorität des Wace'schen Brut.

1 In Wales heifst die Stadt Kaer Loyw, z. B. Brut y Tywys. s. a. 1240; Cair Glovi bei Nennius 49; mit Deutung auf Claudius (f. Gleva) bei Galtr. IV, 15 und Wilh. Gest. Pont. IV, 153. Schon hierin lag ein Anreiz, der Vergangenheit weiter nachzufragen. Aufserdem aber war die Grafschaft von Wales nur durch den Usc getrennt, Caerleon gehörte zu ihr, und im Liber Landavensis S. 27 finden wir Robert in Besitzstreitigkeiten mit der wälschen Abtei. Es ist nicht ausgeschlossen, dafs Robert selbst der Sohn einer Wälschen war, wenn auch nicht der der Nest. Freeman in seiner Polemik gegen die frühere Meinung, Norman Conquest V, 853 schliefst zu Unrecht aus G. R. V, 446 auf französische Abkunft. Wie a Flandrensibus auf Matilda, die Gemahlin Wilhelms, bezieht sich a Francis auf Gisla, die Gemahlin Rollos, entspr. Gesta Regum II, II 2.

G. BAIST. 